Molecllar Physics, 1994, Vol. 81, No. 1, 199-216

\title{
Computer simulation study of a highly polar fluid under the influence of static electric fields
}

\author{
By P. G. KUSALIK \\ Department of Chemistry, Dalhousie University, Halifax, Nova Scotia, B3H 4J3, \\ Canada
}

(Received 11 March 1993; accepted 27 May 1993)

\begin{abstract}
The dielectric response and the electrostriction of polar liquids are certainly important properties of these systems. They also play fundamental roles in processes such as solvation. In this article we report results from a computer simulation study of the behaviour of a highly polar fluid subject to static homogeneous electric fields. Values for three different measures of the dielectric constant are given and their dependences on the field strength analysed. Surprisingly, we find that the integral response and the differential response perpendicular to the field appear to be equal. System size and boundary condition effects are examined; at low fields the behaviour observed reflects that found previously in $\epsilon_{0}$, the zero-field static dielectric constant. As expected at small fields, the dielectric constants and the electrostriction exhibit a simple quadratic dependence upon the field strength. We show that even when very large fields are applied the present dipolar soft-sphere fluid does not demonstrate a phase transition similar to the isotropic-to-nematic transition observed by Wei and Patey at zero field for larger dipole couplings.
\end{abstract}

\section{Introduction}

The phenomena of dielectric saturation and electrostriction are important properties of polar liquids. They are particularly relevant to the theoretical study of solvation and solvation effects in electrolyte solutions where both saturation and electrostriction are believed to be large. Yet, direct observation of either phenomenon in bulk liquids is difficult due to the rather large external fields that are required $[1,2]$. However, computer simulation provides us the opportunity of examining these inherently nonlinear effects in models of polar liquids for arbitrarily large applied electric fields. Such computer experiments can now be performed in a relatively straightforward manner (below we shall see, in fact, that results are more easily obtained at larger fields).

In the past, the determination of the dielectric properties of polar fluids by computer simulation had presented both conceptual and computational difficulties. While many of the conceptual problems have now been at least partially resolved [3-8], the calculation of precise values for the static dielectric constant, $\epsilon_{0}$, can represent a formidable computational task [8-12]. In order to find a more computationally efficient approach, some workers have considered applied field simulations [13-17] as an alternative to the usual equilibrium calculation at zero field which exploits the usual fluctuation formula for $\epsilon_{0}$. The bulk of this work has focused upon simple dipolar fluids where $\epsilon_{0}$ is obtained from the average value of the polarization, $\mathbf{P}$, in the presence of a static homogeneous applied electric field, $\mathbf{E}_{0}$. 
Unfortunately, much of the early work $[14,15]$ suffers from both systematic errors [5] and poor numerical precision. In a recent article [17] static field results along with the transient polarization response of a dipolar system to the instantaneous application of an electric field were reported. From the observed response functions it was concluded that nonlinear effects become important at smaller fields than was previously thought [13-15]. Subsequently, a much more complete investigation [18] of the time-dependent polarization response of the same Stockmayer fluid found no evidence to support the claim in [17].

To our knowledge the work of Adams [15] has been the only previous computer simulation study in which an attempt was made to measure directly electrostriction in a polar liquid. Unfortunately, the precision of his density data was not sufficient to allow Adams to draw any major conclusions.

The present study will examine a highly polar fluid under the influence of static electric fields from several points of view. The particular dipolar soft-sphere fluid being investigated has been studied extensively in previous zero-field work [9, 19]. At non-zero fields it is possible to define at least three different dielectric constants and we will examine how nonlinear (saturation) effects are manifest in these various measures of the polarization response and will explore their dependence upon the field strength. As in our earlier work, system size and boundary condition effects will be closely studied. We will consider whether the average polarization response of the system to small applied fields can be used as a reliable route to the zero-field static dielectric constant. A determination of the electrostriction of the liquid and its dependence upon field strength is another important aspect of the present work.

\section{Dielectric constants at finite fields}

Although various relationships for dielectric constants at finite (non-zero) fields can be found in the literature $[1,8,15,20]$ and in standard references $[21,22]$, nevertheless it will be useful to collect and summarize the resulting expressions here. Since it is possible to define at least three different dielectric constants (each representing a different physical measurement), it will be important that we be able to make clear distinctions between them.

Our starting point is the macroscopic equation

$$
\epsilon_{p} \equiv 1+4 \pi \frac{\mathbf{P}}{\mathbf{E}},
$$

where $\mathbf{P}=\langle\mathbf{M}\rangle / V$ is the polarization, $\mathbf{E}$ is the total internal or Maxwell field, $\langle\mathbf{M}\rangle=\left\langle\Sigma_{i} \boldsymbol{\mu}_{i}\right\rangle$ is the average total dipole moment of the system, and $V$ is the volume. Following Adams $[14,15]$ we will refer to $\epsilon_{p}$ as the integral dielectric constant since it is a measure of the total polarization response of the sample to the applied field. For an isotropic liquid $\mathbf{P}$ and $\mathbf{E}$ will be in the same direction, and hence $\epsilon_{p}$ is a scalar quantity. If $\mathbf{e}$ denotes the direction of $\mathbf{E}$ then we can write

$$
\epsilon_{p}=1+\frac{4 \pi}{V} \frac{\langle\mathbf{M}\rangle \cdot \mathbf{e}}{E} \text {. }
$$

For present purposes it is more convenient to express $\epsilon_{p}$ as a function of the applied electric field, $\mathbf{E}_{0}$, the field that would appear explicitly in the usual hamiltonian for this system. In periodic boundary conditions (PBC), where the system is a macroscopic spherical sample composed of a large number of replicas 
of the basic simulation cell embedded in a continuum with a dielectric constant $\epsilon_{\mathrm{RF}}$, it has now been established $[3,8,10]$ that

$$
\mathbf{E}=\mathbf{E}_{0}-\frac{4 \pi}{V} \frac{\langle\mathbf{M}\rangle}{\left(2 \epsilon_{\mathrm{RF}}+1\right)}=\mathbf{E}_{0}\left(\frac{2 \epsilon_{\mathrm{RF}}+1}{2 \epsilon_{\mathrm{RF}}+\epsilon_{p}}\right) .
$$

We also note that this relationship holds for reaction field boundary conditions (or for any macroscopic spherical sample embedded in a dielectric continuum). Together equations (2) and (3) yield the standard results [8]

$$
\frac{\left(\epsilon_{p}-1\right)\left(2 \epsilon_{\mathrm{RF}}+1\right)}{2 \epsilon_{\mathrm{RF}}+\epsilon_{p}}=\frac{4 \pi}{V} \frac{\langle\mathbf{M} \cdot \mathbf{e}\rangle}{E_{0}} .
$$

In order to explore the dependence of $\epsilon_{p}$ upon the applied field, we expand $\langle\mathbf{M} \cdot \mathbf{e}\rangle$ as a Taylor series about the value $E_{e}$,

$$
\begin{aligned}
\langle\mathbf{M} \cdot \mathbf{e}\rangle_{E_{0}}= & \langle\mathbf{M} \cdot \mathbf{e}\rangle_{E_{e}}+\left(\frac{\partial}{\partial E_{0}}\langle\mathbf{M} \cdot \mathbf{e}\rangle\right)_{E_{e}}\left(E_{0}-E_{e}\right)+\left(\frac{\partial^{2}}{\partial E_{0}^{2}}\langle\mathbf{M} \cdot \mathbf{e}\rangle\right)_{E_{e}} \frac{\left(E_{0}-E_{e}\right)^{2}}{2} \\
& +\left(\frac{\partial^{3}}{\partial E_{0}^{3}}\langle\mathbf{M} \cdot \mathbf{e}\rangle\right)_{E_{e}} \frac{\left(E_{0}-E_{e}\right)^{3}}{6}+\ldots
\end{aligned}
$$

where $E_{e}$ is also assumed to be directed along e and $\langle\cdots\rangle_{E_{0}}$ denotes the ensemble average evaluated in the presence of the field $E_{0}$. From the definition

$$
\langle\mathbf{M} \cdot \mathbf{e}\rangle_{E_{0}}=\frac{\int \mathrm{d} \Gamma(\mathbf{M} \cdot \mathbf{e}) \exp \left[-\beta\left(H_{0}-\mathbf{M} \cdot \mathbf{E}_{0}\right)\right]}{\int \mathrm{d} \Gamma \exp \left[-\beta\left(H_{0}-\mathbf{M} \cdot \mathbf{E}_{0}\right)\right]},
$$

explicit expressions for the partial derivatives appearing in equation (5) can be obtained and it can be rewritten as

$$
\langle\mathbf{M} \cdot \mathbf{e}\rangle_{E_{0}}=a_{0}+a_{1} \Delta E_{0}+a_{2} \Delta E_{0}^{2}+a_{3} \Delta E_{0}^{3}+\ldots,
$$

where

$$
\begin{aligned}
\Delta E_{0} & =E_{0}-E_{e}, \\
a_{0} & =\langle\mathbf{M} \cdot \mathbf{e}\rangle_{E_{e}}, \\
a_{1} & =\beta\left(\left\langle(\mathbf{M} \cdot \mathbf{e})^{2}\right\rangle_{E_{e}}-\langle\mathbf{M} \cdot \mathbf{e}\rangle_{E_{e}}^{2}\right), \\
a_{2} & =\frac{\beta^{2}}{2}\left(\left\langle(\mathbf{M} \cdot \mathbf{e})^{3}\right\rangle_{E_{e}}-3\left\langle(\mathbf{M} \cdot \mathbf{e})^{2}\right\rangle_{E_{e}}\langle\mathbf{M} \cdot \mathbf{e}\rangle_{E_{e}}+2\langle\mathbf{M} \cdot \mathbf{e}\rangle_{E_{e}}^{3}\right), \\
a_{3} & =\frac{\beta^{3}}{6}\left(\begin{array}{c}
\left\langle(\mathbf{M} \cdot \mathbf{e})^{4}\right\rangle_{E_{e}}-4\left\langle(\mathbf{M} \cdot \mathbf{e})^{3}\right\rangle_{E_{e}}\langle\mathbf{M} \cdot \mathbf{e}\rangle_{E_{e}}-3\left\langle(\mathbf{M} \cdot \mathbf{e})^{2}\right\rangle_{E_{e}}^{2} \\
+12\left\langle(\mathbf{M} \cdot \mathbf{e})^{2}\right\rangle_{E_{e}}\langle\mathbf{M} \cdot \mathbf{e}\rangle_{E_{e}}^{2}-6\langle\mathbf{M} \cdot \mathbf{e}\rangle_{E_{e}}^{4}
\end{array}\right),
\end{aligned}
$$

and $\beta=1 / k T$.

If we now restrict ourselves to the case $E_{e}=0$, the probability of any particular value of the polarization is independent of its direction, and therefore

$$
\left\langle(\mathbf{M} \cdot \mathbf{e})^{n}\right\rangle_{E_{e}=0}=0, \quad \text { for } n \text { odd } .
$$


It immediately follows then that

$$
\begin{aligned}
& a_{0}=a_{2}=0, \\
& a_{1}=\frac{\beta}{3}\left\langle M^{2}\right\rangle_{0},
\end{aligned}
$$

and

$$
a_{3}=\frac{\beta^{3}}{90}\left(3\left\langle M^{4}\right\rangle_{0}-5\left\langle M^{2}\right\rangle_{0}^{2}\right)
$$

where $\langle\cdots\rangle_{0}$ indicates the zero-field ensemble average. Thus, in the limit of very small applied fields we can insert equations (9), $(7 a)$ and $(7 b)$ into equation (4) to obtain

$$
\frac{\left(\epsilon_{p}-1\right)\left(2 \epsilon_{\mathrm{RF}}+1\right)}{2 \epsilon_{\mathrm{RF}}+\epsilon_{p}}=\frac{4 \pi}{V}\left[\frac{\beta}{3}\left\langle M^{2}\right\rangle_{0}+\frac{\beta^{3}}{90}\left(3\left\langle M^{4}\right\rangle_{0}-5\left\langle M^{2}\right\rangle_{0}^{2}\right) E_{0}^{2}\right] .
$$

Rearranging equation (10) and defining the zero-field dielectric constant (still following previous workers $[8,10])$,

$$
\epsilon_{0} \equiv \frac{1+2 \epsilon_{\mathrm{RF}}+2 \epsilon_{\mathrm{RF}} \frac{4 \pi \beta}{3 V}\left\langle M^{2}\right\rangle_{0}}{1+2 \epsilon_{\mathrm{RF}}-\frac{4 \pi \beta}{3 V}\left\langle M^{2}\right\rangle_{0}}
$$

it is easily shown that

$$
\epsilon_{p}=\epsilon_{0}+\left(\frac{\epsilon_{0}+2 \epsilon_{\mathrm{RF}}}{2 \epsilon_{\mathrm{RF}}+1}\right)^{2}\left[\frac{4 \pi}{V} \frac{\beta^{3}}{90}\left(3\left\langle M^{4}\right\rangle_{0}-5\left\langle M^{2}\right\rangle_{0}^{2}\right)\right] E_{0}^{2},
$$

which will be valid in the limit of small $E_{0}$. Three special cases of equation (12) are of particular interest, namely,

$$
\begin{aligned}
& \text { when } \epsilon_{\mathrm{RF}}=\infty, \quad \text { then } \epsilon_{p}=\epsilon_{0}+\frac{4 \pi}{V} a_{3} E_{0}^{2}, \\
& \text { when } \epsilon_{\mathrm{RF}}=\epsilon_{0}, \quad \text { then } \epsilon_{p}=\epsilon_{0}+\frac{4 \pi}{V}\left(\frac{3 \epsilon_{0}}{2 \epsilon_{0}+1}\right)^{2} a_{3} E_{0}^{2}, \\
& \text { and when } \epsilon_{\mathrm{RF}}=1, \quad \text { then } \epsilon_{p}=\epsilon_{0}+\frac{4 \pi}{V}\left(\frac{\epsilon_{0}+2}{3}\right)^{2} a_{3} E_{0}^{2} .
\end{aligned}
$$

Equation (12) can also be expressed in terms of the Maxwell field, $E$; using equation (3) one obtains that

$$
\epsilon_{p}=\epsilon_{0}+\left(\frac{\epsilon_{0}+2 \epsilon_{\mathrm{RF}}}{2 \epsilon_{\mathrm{RF}}+1}\right)^{2}\left(\frac{\epsilon_{p}+2 \epsilon_{\mathrm{RF}}}{2 \epsilon_{\mathrm{RF}}+1}\right)^{2}\left[\frac{4 \pi}{V} \frac{\beta^{3}}{90}\left(3\left\langle M^{4}\right\rangle_{0}-5\left\langle M^{2}\right\rangle_{0}^{2}\right)\right] E^{2} .
$$

For $\epsilon_{\mathrm{RF}}=1$ and to leading order in $E$ (when $\epsilon_{p}$ can be replaced by $\epsilon_{0}$ ), equation (14) agrees with the much earlier work of Buckingham [1] and Coffey and Scaife (see equation (13) of [20]). We remark that the second term in equations (12), (13) and (14) represent the leading-order nonlinear terms in the polarization response of the system.

It is often only the linear polarization response of the sample to the applied field which is desired, and hence a second definition,

$$
\epsilon_{f} \equiv 1+\frac{4 \pi}{V} \frac{\partial}{\partial E}\langle\mathbf{M} \cdot \mathbf{e}\rangle=1+\frac{4 \pi}{V}\left(\frac{\partial E_{0}}{\partial E}\right) \frac{\partial}{\partial E_{0}}\langle\mathbf{M} \cdot \mathbf{e}\rangle,
$$


is used for what is known as the incremental [1,21] or differential [15] dielectric constant. Employing expressions analogous to equations (3) and $(7 d)$ one immediately has that for sufficiently (infinitesimally) small $E_{0}$, such that there is no coupling between the direction and magnitude of $\mathbf{M}$,

$$
\frac{\left(\epsilon_{f}-1\right)\left(2 \epsilon_{\mathrm{RF}}+1\right)}{2 \epsilon_{\mathrm{RF}}+\epsilon_{f}}=\frac{4 \pi \beta}{3 V}\left(\left\langle M^{2}\right\rangle-\left\langle\langle M\rangle^{2}\right)=\frac{4 \pi \beta}{3 V}\left\langle M^{2}\right\rangle\right.
$$

where the second equality follows from the fact that $\langle M\rangle=0$. Equation (16) represents the usual fluctuation formula for the dielectric constant and is clearly equivalent to equation (11).

However, at finite fields the system will no longer be isotropic and we would expect its response to depend upon the relative orientations of the probe and polarizing fields. In the presence of the applied field $\mathbf{E}_{0}$, it is then possible to define two unique incremental dielectric constants, one measuring the differential response parallel to $\mathbf{E}_{0}$,

$$
\epsilon_{\|} \equiv 1+\frac{4 \pi}{V} \frac{\partial}{\partial E}\left\langle M_{\|}\right\rangle_{E_{0}}
$$

and one measuring the differential response perpendicular to $\mathbf{E}_{0}$,

$$
\epsilon_{\perp} \equiv 1+\frac{4 \pi}{V} \frac{\partial}{\partial E}\left\langle M_{\perp}\right\rangle_{E_{0}} .
$$

It is straightforward to show that for very small probe fields

$$
\frac{\left(\epsilon_{\|}-1\right)\left(2 \epsilon_{\mathrm{RF}}+1\right)}{2 \epsilon_{\mathrm{RF}}+\epsilon_{\|}}=\frac{4 \pi \beta}{V}\left(\left\langle M_{\|}{ }^{2}\right\rangle_{E_{0}}-\left\langle M_{\|}\right\rangle_{E_{0}}^{2}\right)
$$

and

$$
\frac{\left(\epsilon_{\perp}-1\right)\left(2 \epsilon_{\mathrm{RF}}+1\right)}{2 \epsilon_{\mathrm{RF}}+\epsilon_{\perp}}=\frac{4 \pi \beta}{V}\left\langle M_{\perp}^{2}\right\rangle_{E_{0}}
$$

where we have taken advantage of the fact that $\left\langle M_{\perp}\right\rangle_{E_{0}}=0$.

\section{Model and simulation methodology}

As a detailed description of the present model and general simulation methodology can be found elsewhere $[9,23,24]$, here we only briefly outline some key points.

In the current investigation we employ the same soft-sphere model which has been extensively studied in earlier work $[9,19]$. Its pair potential can be expressed as

$$
u(12)=u_{\mathrm{ss}}(r)+u_{\mathrm{dd}}(12),
$$

in which

$$
u_{s s}(r)=4 \epsilon_{s s}\left(\frac{\sigma}{r}\right)^{12}
$$

is just the soft-sphere potential, and

$$
u_{\mathrm{dd}}(12)=-3\left(\boldsymbol{\mu}_{1} \cdot \mathbf{r}\right)\left(\boldsymbol{\mu}_{2} \cdot \mathbf{r}\right) / r^{5}+\boldsymbol{\mu}_{1} \cdot \boldsymbol{\mu}_{2} / r^{3}
$$

is the dipole-dipole interaction, where $\mu_{1}$ and $\mu_{2}$ are dipole moments of particles 1 and 2 , and $\mathbf{r}=\mathbf{r}_{2}-\mathbf{r}_{1}$ is the separation vector. In the presence of the homogeneous 
applied field $\mathbf{E}_{0}$, the total energy of particle $i$ becomes

$$
U_{i}^{\prime}=U_{i}-\boldsymbol{\mu}_{i} \cdot \mathbf{E}_{0},
$$

where $U_{i}$ is just its total energy with the field off. Thus, $E_{0}$ is the applied field actually experienced by each particle; in this study it is always assumed to be directed along the $z$ axis.

Cubic samples of $32,108,256$ and 500 particles were examined in the present calculations. Periodic boundary conditions were employed with the required lattice sums being performed using the Ewald summation technique $[4,10,24]$. As in previous work $[9,19]$ care was taken in choosing the truncation points and the value for the unitless convergence parameter $\alpha$. In our simulations the real space sum was carried over all nearest images, while in reciprocal space only those vectors $\mathbf{n}$ for which $n^{2} \leqslant n_{\max }^{2}$ were included. The values of $n_{\max }^{2}$ and $\alpha$ used here were the same as those employed in our earlier investigations of this system and can be found in table IV of [9].

Molecular dynamics simulations were carried out at constant temperature with the aid of a Gaussian thermostat [23,24]. In order to study electrostriction, constant pressure conditions were maintained by a Nosé barostat [23-25]. The resulting equations of motion can be written as

$$
\begin{aligned}
& \dot{\mathbf{p}}_{i}=\mathbf{F}_{i}-\gamma \mathbf{p}_{i}-\xi \mathbf{p}_{i}, \\
& \dot{\boldsymbol{\lambda}}_{i}=\mathbf{T}_{i}-\gamma \boldsymbol{\lambda}_{i},
\end{aligned}
$$

and

$$
\dot{\mathbf{q}}_{i}=\mathbf{p}_{i} / m_{i}+\xi \mathbf{q}_{i},
$$

where the equations governing the motions of the orientational coordinates (the quaterions $[24,26])$ remain unchanged. In equations $(20), \mathbf{p}_{i}$ and $\boldsymbol{\lambda}_{i}$ are, respectively, the linear and principal angular momenta of particle $i, \mathbf{q}_{i}$ denotes its position, $\mathbf{F}_{i}$ is the force and $\mathbf{T}_{i}$ is the torque acting on particle $i$. For these isokinetic equations of motion,

$$
\gamma=\frac{\sum_{i} \mathbf{p}_{i} \cdot \mathbf{F}_{i} / m_{i}+\sum_{i} \boldsymbol{\lambda}_{i} \cdot \mathbf{T}_{i} / I_{i}-\xi \sum_{i} p_{i}^{2} / m_{i}}{\sum_{i} p_{i}^{2} / m_{i}+\sum_{i} \lambda_{i}^{2} / I_{i}}
$$

is the Gaussian multiplier which maintains a constant total kinetic energy, where $m_{i}$ and $I_{i}$ are, respectively, the mass and principal moment of inertia. For the Nose barostat the time evolution of the dilation rate, $\xi$, is given by $[23,24]$

$$
\dot{\xi}=\frac{1}{Q}\left(P-P^{0}\right)
$$

in which $P$ and $P^{0}$ are, respectively, the instantaneous and desired pressures of the system, and $Q$ is an adjustable parameter (the 'piston mass') associated with the extended system. If we let $L$ denote the length of our cubic simulation cell, then it is clear from our equations of motion that

$$
\dot{L}=\xi L,
$$

from which it immediately follows that the evolution of the volume and the density will be governed by the equations

$$
\dot{V}=3 \xi V
$$


and

$$
\dot{\rho}=-3 \xi \rho,
$$

respectively. Equations (23) imply that our momenta $\mathbf{p}_{i}$ are peculiar momenta, with $\xi \mathbf{q}_{i}$ being the local 'streaming' velocities of our particles.

In our calculations the equations of motion were integrated using a fourth-order Gear algorithm and a timestep, $\Delta t$, of $0.0025\left(m \sigma^{2} / \epsilon_{\mathrm{ss}}\right)^{\frac{1}{2}}$. Runs varied in lengths from $1 \times 10^{4}$ to $4 \times 10^{5}$ timesteps, the longest simulations being performed at the smallest fields. Each run was preceded by a suitable equilibration period of typically 5000 20000 timesteps, the starting configuration usually coming from a wellequilibrated system at a higher field.

\section{Results and discussion}

In our calculations and in the following discussion it is convenient to express all parameters in reduced units. The particular dipolar soft-sphere fluid we have considered can be characterized at constant volume and zero field by a reduced density,

$$
\rho^{*}=\rho \sigma^{3}=0 \cdot 8,
$$

a reduced temperature,

$$
T^{*}=k T / \epsilon_{\mathrm{ss}}=1 \cdot 35,
$$

and a reduced dipole moment,

$$
\mu^{*}=\left(\mu^{2} / \epsilon_{\mathrm{ss}} \sigma^{3}\right)^{1 / 2}=2 \cdot 0 .
$$

As mentioned above, this dipolar soft-sphere system has been the subject of earlier simulation work $[9,19]$. We point out that unlike a Stockmayer fluid at the same state point, the dipolar soft-sphere fluid has a positive pressure. In the present study this fluid is examined at constant pressure in the presence of static homogeneous electric fields, $E_{0}^{*}=E_{0}\left(\sigma^{3} / \epsilon_{\mathrm{ss}}\right)^{\frac{1}{2}}$, ranging in value from 0.025 to $1 \cdot 0$. For values of $\sigma$ and $\epsilon_{\mathrm{ss}}$ roughly corresponding to those used in water-like models, these fields would span a range of about $10^{6}-10^{8} \mathrm{~V} \mathrm{~cm}^{-1}$.

Tables 1, 2, 3 and 4 summarized the simulations carried out for systems of 32, 108, 256 and 500 particles, respectively. In each case sets of runs were performed in 'tin-foil' (i.e., $E=E_{0}$ ) boundary conditions, as well as with $\epsilon_{\mathrm{RF}}=85$ (which is approximately the value of $\epsilon_{0}$ ). For $N=108$, an additional set was studied at $\epsilon_{\mathrm{RF}}=15$. We remark that 'tin-foil' boundary conditions have tranditionally been viewed as referring to $\epsilon_{\mathrm{RF}}=\infty$ when applied to zero-field calculations. However, this interpretation must be modified when considering applied field simulations where these boundary conditions should be expressed as the limit of $\epsilon_{\mathrm{RF}} \rightarrow \infty$ (then, in order to obtain the appropriate value of $E_{0}$ we can imagine applying an external field which grows as rapidly as $\epsilon_{\mathrm{RF}}$ ). Thus, our calculations can be seen as being carried out at some large but finite value of $\epsilon_{\mathrm{RF}}$, which on a computer of limited numerical accuracy becomes indistinguishable from $\epsilon_{\mathrm{RF}}=\infty$. Values of $Q$ were chosen to allow the fluctuations in the pressure to occur over reasonable time-scales (of the order of tens to hundreds of timesteps); short test calculations confirmed that the quantities of interest were invariant to the exact choice for $Q$. The pressures were chosen so as to recover reduced densities of 0.8 at zero field. Table 2 contains results from a test run for $N=108$ and $\epsilon_{\mathrm{RF}}=\infty$ at $E_{0}^{*}=0$; 
Table 1. Constant pressure results for 32-particle dipolar soft-sphere systems at $T^{*}=1.35$ and $\mu^{*}=2 \cdot 0$. Estimates of the uncertainties for the dielectric constants are given in parentheses.

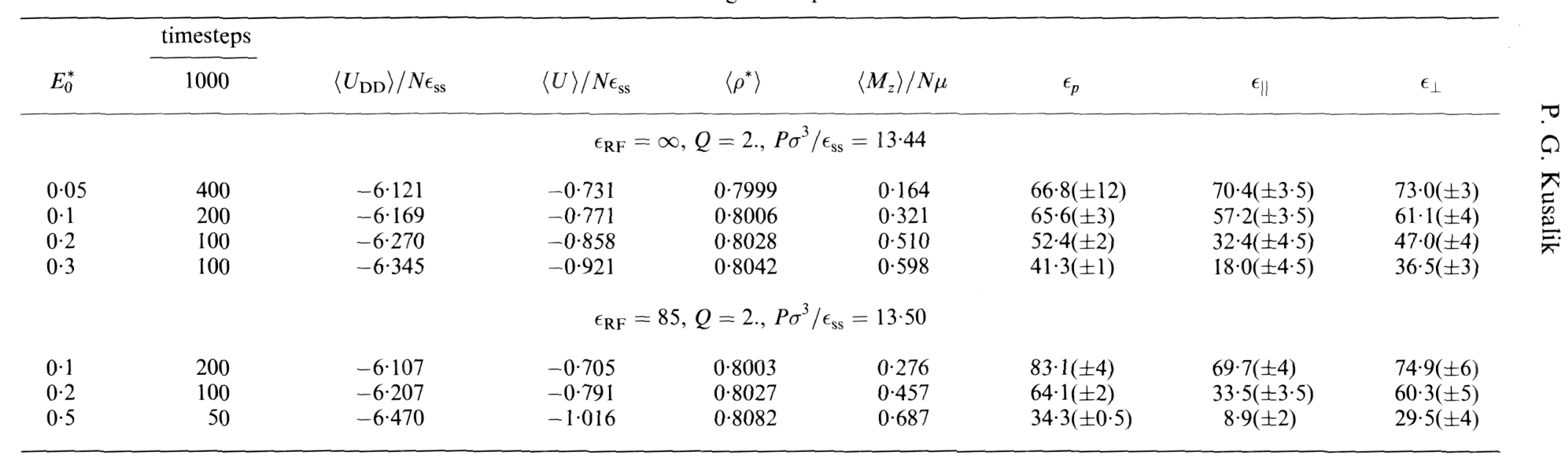


Table 2. Constant pressure results for 108-particle dipolar soft-sphere systems at $T^{*}=1 \cdot 35$ and $\mu^{*}=2 \cdot 0$. Estimates of the uncertainties for the dielectric constants are given in parentheses.

\begin{tabular}{|c|c|c|c|c|c|c|c|c|}
\hline \multirow[b]{2}{*}{$E_{0}^{*}$} & timesteps & \multirow[b]{2}{*}{$\left\langle U_{\mathrm{DD}}\right\rangle / N \epsilon_{\mathrm{ss}}$} & \multirow[b]{2}{*}{$\langle U\rangle / N \epsilon_{\mathrm{ss}}$} & \multirow[b]{2}{*}{$\left\langle\rho^{*}\right\rangle$} & \multirow[b]{2}{*}{$\left\langle M_{z}\right\rangle / N \mu$} & \multirow[b]{2}{*}{$\epsilon_{p}$} & \multirow[b]{2}{*}{$\epsilon_{\|}$} & \multirow[b]{2}{*}{$\epsilon_{\perp}$} \\
\hline & 1000 & & & & & & & \\
\hline \multicolumn{9}{|c|}{$\epsilon_{\mathrm{RF}}=\infty, Q=1 ., P \sigma^{3} / \epsilon_{\mathrm{ss}}=13.40$} \\
\hline $0 \cdot 0$ & 300 & -6.057 & -0.693 & $0 \cdot 7998$ & - & - & $86 \cdot 5( \pm 4)$ & $86 \cdot 5( \pm 4)$ \\
\hline 0.025 & 400 & $-6 \cdot 059$ & -0.696 & $0 \cdot 8001$ & $0 \cdot 112$ & $91 \cdot 3( \pm 8)$ & $76 \cdot 8( \pm 3)$ & $79 \cdot 5( \pm 6)$ \\
\hline $0 \cdot 05$ & 200 & $-6 \cdot 084$ & -0.716 & $0 \cdot 8003$ & $0 \cdot 206$ & $84 \cdot 0( \pm 4)$ & $69 \cdot 7( \pm 4)$ & $82 \cdot 4( \pm 3)$ \\
\hline $0 \cdot 1$ & 100 & $-6 \cdot 148$ & -0.771 & $0 \cdot 8014$ & 0.353 & $72 \cdot 1( \pm 5)$ & $47 \cdot 5( \pm 3)$ & $74 \cdot 8( \pm 4)$ \\
\hline $0 \cdot 2$ & 100 & $-6 \cdot 263$ & $-0 \cdot 868$ & $0 \cdot 8038$ & 0.536 & $55 \cdot 2( \pm 2)$ & $24 \cdot 6( \pm 1)$ & $51 \cdot 9( \pm 3 \cdot 5)$ \\
\hline $0 \cdot 3$ & 50 & $-6 \cdot 352$ & $-0 \cdot 943$ & $0 \cdot 0850$ & 0.620 & $42 \cdot 8( \pm 1)$ & $15 \cdot 3( \pm 1)$ & $41 \cdot 1( \pm 4)$ \\
\hline 0.5 & 50 & $-6 \cdot 475$ & $-1 \cdot 050$ & $0 \cdot 8083$ & 0.712 & $29 \cdot 9( \pm 0 \cdot 8)$ & $6 \cdot 8( \pm 0 \cdot 5)$ & $24 \cdot 9( \pm 2 \cdot 5)$ \\
\hline 0.75 & 50 & $-6 \cdot 571$ & $-1 \cdot 129$ & $0 \cdot 8097$ & $0 \cdot 765$ & $21 \cdot 8( \pm 0 \cdot 5)$ & $4 \cdot 2( \pm 0 \cdot 3)$ & $20 \cdot 6( \pm 1)$ \\
\hline $1 \cdot 0$ & 25 & $-6 \cdot 645$ & $-1 \cdot 195$ & $0 \cdot 8117$ & $0 \cdot 799$ & $17 \cdot 3( \pm 0 \cdot 2)$ & $3 \cdot 5( \pm 0 \cdot 3)$ & $16 \cdot 8( \pm 1)$ \\
\hline \multicolumn{9}{|c|}{$\epsilon_{\mathrm{RF}}=85, Q=1 ., P \sigma^{3} / \epsilon_{\mathrm{ss}}=13.40$} \\
\hline 0.05 & 400 & $-6 \cdot 052$ & -0.691 & $0 \cdot 8001$ & $0 \cdot 149$ & $93 \cdot 1( \pm 7)$ & $91 \cdot 9( \pm 5)$ & $86 \cdot 2( \pm 6)$ \\
\hline $0 \cdot 1$ & 200 & $-6 \cdot 087$ & $-0 \cdot 720$ & $0 \cdot 8006$ & $0 \cdot 276$ & $83 \cdot 3( \pm 5)$ & $56 \cdot 5( \pm 3)$ & $83 \cdot 6( \pm 5)$ \\
\hline $0 \cdot 2$ & 100 & $-6 \cdot 185$ & $-0 \cdot 803$ & $0 \cdot 8025$ & $0 \cdot 449$ & $62 \cdot 7( \pm 2)$ & $35 \cdot 0( \pm 2)$ & $66 \cdot 1( \pm 5)$ \\
\hline 0.5 & 50 & $-6 \cdot 429$ & $-1 \cdot 011$ & $0 \cdot 8072$ & 0.682 & $34 \cdot 0( \pm 1)$ & $8 \cdot 9( \pm 1)$ & $30 \cdot 4( \pm 3)$ \\
\hline $1 \cdot 0$ & 25 & $-6 \cdot 625$ & $-1 \cdot 172$ & 0.8103 & 0.791 & $18 \cdot 8( \pm 0 \cdot 5)$ & $3 \cdot 3( \pm 0 \cdot 5)$ & $18 \cdot 1( \pm 1 \cdot 5)$ \\
\hline \multicolumn{9}{|c|}{$\epsilon_{\mathrm{RF}}=15, Q=1 ., P \sigma^{3} / \epsilon_{\mathrm{ss}}=13 \cdot 40$} \\
\hline $0 \cdot 1$ & 400 & $-6 \cdot 025$ & -0.666 & $0 \cdot 7994$ & $0 \cdot 118$ & $101 \cdot 1( \pm 10)$ & $115 \cdot 8( \pm 20)$ & $100 \cdot 8( \pm 10)$ \\
\hline $0 \cdot 2$ & 200 & $-6 \cdot 059$ & $-0 \cdot 695$ & $0 \cdot 7999$ & $0 \cdot 234$ & $97 \cdot 9( \pm 6)$ & $68 \cdot 4( \pm 8)$ & $96 \cdot 0( \pm 12)$ \\
\hline $0 \cdot 3$ & 100 & $-6 \cdot 095$ & -0.727 & $0 \cdot 8008$ & $0 \cdot 336$ & $83 \cdot 9( \pm 4)$ & $60 \cdot 2( \pm 8)$ & $64 \cdot 4( \pm 10)$ \\
\hline 0.5 & 50 & $-6 \cdot 215$ & -0.829 & $0 \cdot 8032$ & $0 \cdot 512$ & $63 \cdot 1( \pm 2)$ & $34 \cdot 1( \pm 5)$ & $81 \cdot 0( \pm 14)$ \\
\hline $0 \cdot 8$ & 50 & $-6 \cdot 387$ & -0.975 & $0 \cdot 8066$ & $0 \cdot 664$ & $37 \cdot 8( \pm 1)$ & $11 \cdot 8( \pm 3)$ & $34 \cdot 1( \pm 6)$ \\
\hline
\end{tabular}


Table 3. Constant pressure results for 256-particle dipolar soft-sphere systems at $T^{*}=1 \cdot 35$ and $\mu^{*}=2 \cdot 0$. Estimates of the uncertainties for the dielectric constants are given in parentheses.

\begin{tabular}{|c|c|c|c|c|c|c|c|c|}
\hline \multirow[b]{2}{*}{$E_{0}^{*}$} & timesteps & \multirow[b]{2}{*}{$\left\langle U_{\mathrm{DD}}\right\rangle / N \epsilon_{\mathrm{ss}}$} & \multirow[b]{2}{*}{$\langle U\rangle / N \epsilon_{\mathrm{ss}}$} & \multirow[b]{2}{*}{$\left\langle\rho^{*}\right\rangle$} & \multirow[b]{2}{*}{$\left\langle M_{z}\right\rangle / N \mu$} & \multirow[b]{2}{*}{$\epsilon_{p}$} & \multirow[b]{2}{*}{$\epsilon_{\|}$} & \multirow[b]{2}{*}{$\epsilon_{\perp}$} \\
\hline & 1000 & & & & & & & \\
\hline \multicolumn{9}{|c|}{$\epsilon_{\mathrm{RF}}=\infty, Q=1 ., P \sigma^{3} / \epsilon_{\mathrm{ss}}=13.45$} \\
\hline 0.025 & 400 & -6.072 & -0.688 & 0.7999 & $0 \cdot 122$ & $99 \cdot 3( \pm 6)$ & $78 \cdot 6( \pm 3)$ & $87 \cdot 4( \pm 7)$ \\
\hline 0.05 & 200 & -6.091 & $-0 \cdot 706$ & 0.8005 & 0.222 & $90 \cdot 2( \pm 4)$ & $76 \cdot 0( \pm 4 \cdot 5)$ & $82 \cdot 8( \pm 6)$ \\
\hline $0 \cdot 1$ & 100 & $-6 \cdot 148$ & $-0 \cdot 754$ & 0.8018 & $0 \cdot 365$ & $74 \cdot 4( \pm 2)$ & $55 \cdot 4( \pm 4 \cdot 5)$ & $70 \cdot 3( \pm 4)$ \\
\hline $0 \cdot 2$ & 100 & $-6 \cdot 271$ & $-0 \cdot 858$ & $0 \cdot 8039$ & $0 \cdot 532$ & $54 \cdot 7( \pm 1)$ & $24 \cdot 9( \pm 1 \cdot 5)$ & $61 \cdot 5( \pm 5)$ \\
\hline \multicolumn{9}{|c|}{$\epsilon_{\mathrm{RF}}=85, Q=1 ., P \sigma^{3} / \epsilon_{\mathrm{ss}}=13.46$} \\
\hline 0.05 & 200 & -6.069 & -0.684 & 0.8002 & $0 \cdot 153$ & $97 \cdot 1( \pm 10)$ & $95 \cdot 3( \pm 5)$ & $106 \cdot 8( \pm 12)$ \\
\hline $0 \cdot 1$ & 100 & $-6 \cdot 112$ & $-0 \cdot 720$ & 0.8008 & $0 \cdot 281$ & $85 \cdot 6( \pm 4)$ & $76 \cdot 5( \pm 8)$ & $75 \cdot 9( \pm 7)$ \\
\hline 0.2 & 50 & $-6 \cdot 216$ & $-0 \cdot 810$ & 0.8029 & 0.471 & $66 \cdot 8( \pm 2)$ & $37 \cdot 8( \pm 4)$ & $68 \cdot 2( \pm 6)$ \\
\hline 0.5 & 30 & $-6 \cdot 442$ & $-1 \cdot 002$ & 0.8076 & 0.686 & $34 \cdot 3( \pm 1)$ & $10 \cdot 2( \pm 1)$ & $34 \cdot 0( \pm 3)$ \\
\hline
\end{tabular}


Table 4. Constant pressure results for 500 -particle dipolar soft-sphere systems at $T^{*}=1 \cdot 35$ and $\mu^{*}=2 \cdot 0$. Estimates of the uncertainties for the dielectric constants are given in parentheses.

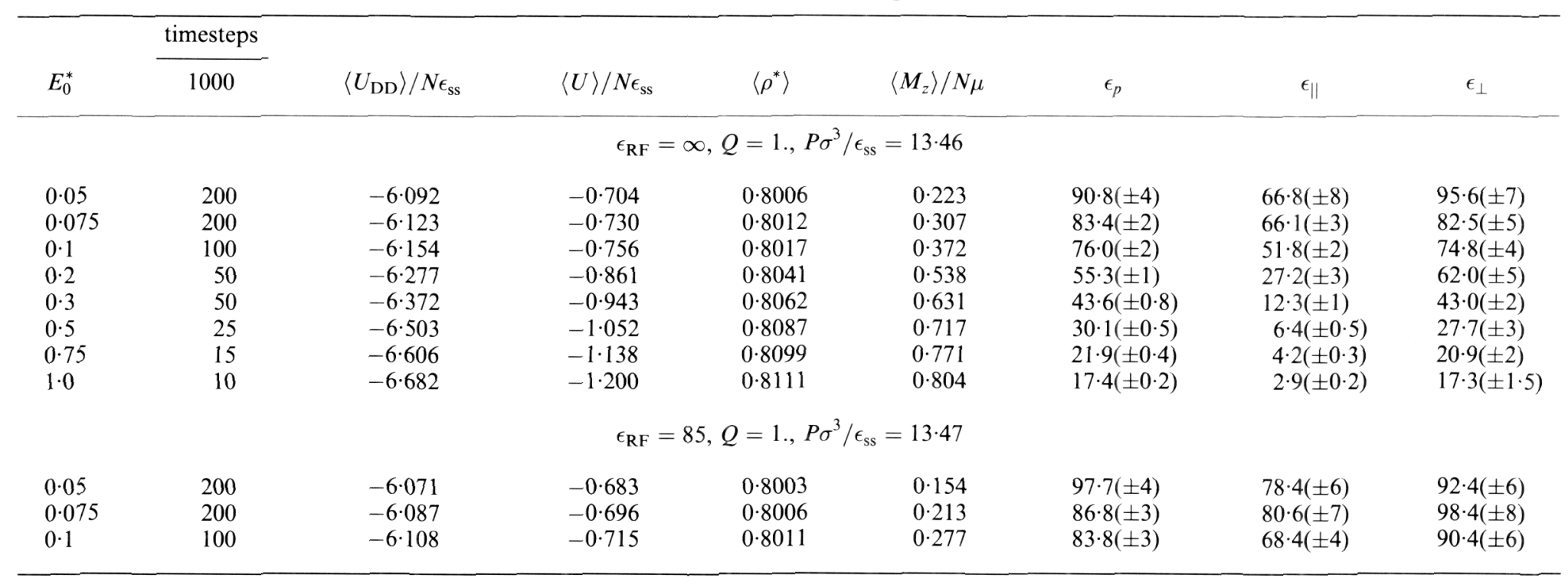


we find excellent agreement between it and our previous constant volume calculations [9].

Estimates for our statistical uncertainties in average quantities were usually determined by dividing each run into several blocks and computing the standard deviation of the mean. Values for the uncertainties in the dielectric constants are given in tables 1-4. Estimates for the error bars for the average energies range from \pm 0.02 for the shortest runs (high fields) and smallest systems, to \pm 0.003 for the longest runs and largest systems. Similarly, the uncertainties in the average densities range from \pm 0.001 to \pm 0.00015 .

In tables 1-4 we have summarized results for the average dipole-dipole energy, $\left\langle U_{\mathrm{DD}}\right\rangle / N \epsilon_{\mathrm{ss}}$, the average total configurational energy, $\langle U\rangle / N \epsilon_{\mathrm{ss}}$, and the average reduced density, $\left\langle\rho^{*}\right\rangle$. The average polarization we have reported as $\left\langle M_{z}\right\rangle / N_{\mu}$, which has a limiting value of $1 \cdot 0$ for a totally saturated (aligned) system. The thermodynamic properties exhibit small but clear tends with increasing field strength. The changes in the total energy are dominated by the increasing magnitude of the dipole-dipole contribution. Electrostriction of the fluid is observed, that is the density is found to increase with the applied field. The dependence of $\left\langle\rho^{*}\right\rangle$ upon $E_{0}^{*}$ will be examined in detail below. We also see from tables 1-4 that even at the smallest fields studied, the samples are still more than $10 \%$ saturated. For the largest fields included in tables 1-4, the polarization is approximately $80 \%$ of its maximum value, indicating a rather high degree of orientational ordering (or dipole alignment). As one would expect, we find that the measured polarization response is dependent upon $\epsilon_{\mathrm{RF}}$; for a given field strength $\left\langle M_{z}\right\rangle$ becomes smaller with decreasing $\epsilon_{\mathrm{RF}}$.

Also recorded in tables 1-4 are results for three distinct measures of the dielectric response, the integral dielectric constant, $\epsilon_{p}$, as given by equation (4), and the two differential (or incremental) dielectric constants, $\epsilon_{\|}$and $\epsilon_{\perp}$, as determined from equations (18). In general, all three dielectric constants decrease with increasing $E_{0}^{*}$. We find that the largest uncertainties for the dielectric constants are still observed at the lowest fields despite significantly longer run lengths. A comparison with zero-field calculations [9] indicates that applied field simulations at very small fields are not as computationally efficient as the usual equilibrium fluctuation approach to $\epsilon_{0}$.

The dependence of the dielectric constants $\epsilon_{p}, \epsilon_{\|}$and $\epsilon_{\perp}$ upon the strength of the applied electric field is examined in figure 1 for samples of 108 and 500 dipolar softspheres. Several features in figure 1 are noteworthy. The dielectric constants all decrease in value with increasing field as a result of saturation, although the behaviour appears more confused at small fields due to larger uncertainties. Again, we would conclude that simulations at small applied fields do not represent a superior route to $\epsilon_{0}$. We find that the response as measured by $\epsilon_{\|}$decreases most rapidly, its values always lying below those of $\epsilon_{\perp}$ and $\epsilon_{p}$. This is perhaps not surprising since we would expect the differential response to be smaller parallel to the direction of alignment than perpendicular to it. A more striking result is that to within our uncertainties $\epsilon_{\perp}=\epsilon_{p}$, independent of field strength. Why this differential and the integral dielectric constants should be equal is not obvious. Adams proposed that (see equation (12) of [15])

$$
\frac{\left(\epsilon_{\perp}-1\right)^{2}}{\left(\epsilon_{\|}-1\right)}=\left(\epsilon_{p}-1\right)
$$




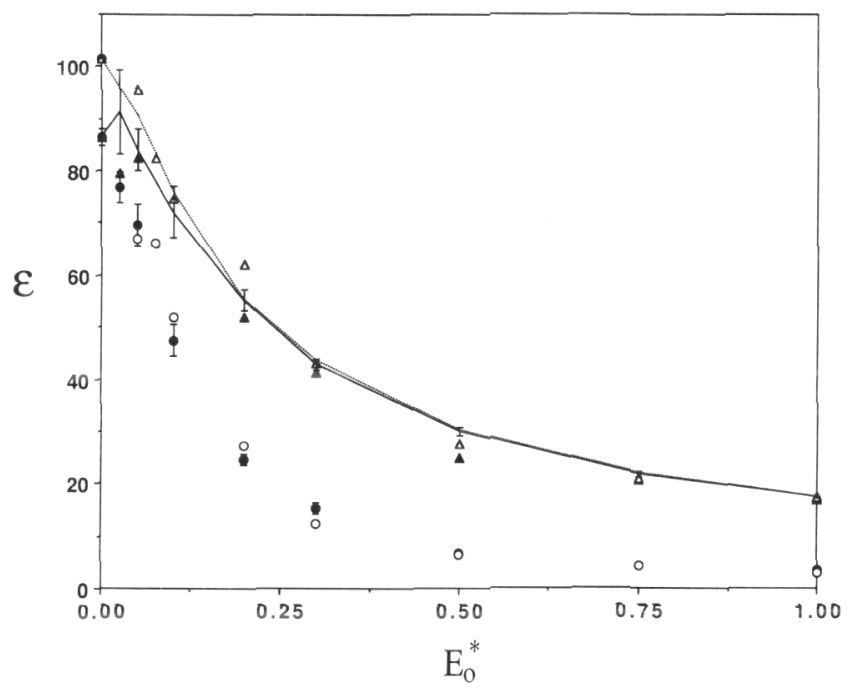

Figure 1. Dependence of $\epsilon_{p}, \epsilon_{\|}$and $\epsilon_{\perp}$ upon the applied field for systems of 108 and 500 particles at $\epsilon_{\mathrm{RF}}=\infty$. The full circles, full triangles, and solid line represent, respectively, iesults for $\epsilon_{\|}, \epsilon_{\perp}$ and $\epsilon_{p}$ for $N=108$, while the open circles, open triangles, and dotted line are values recorded for $N=500$. Sample error bars have been included. The points at $E_{0}^{*}=0$ have been taken from [9].

unfortunately, he did not give a justification or source for this relationship. It is clear from the behaviour exhibited in figure 1 and from the numerical values in tables 1-4 that this equality does not hold. Curiously, however, if we examine values for the left-hand side of equation (25) as a function of $E_{0}^{*}$ for the systems studied, we discover that they are roughly constant and perhaps suspiciously close to $\epsilon_{0}$. Again, a justification for this apparent behaviour is not immediately obvious.

We see from figure 1 that each of the measures of the dielectric response at finite field is consistent with our previously determined zero-field points (as obtained in [9] from equation (11)). Moreover, the system size dependence evident in $\epsilon_{0}$ is reflected in the small field behaviour of the dielectric constants, most notably for $\epsilon_{p}$. Yet, as the applied electric field becomes larger and the saturation increases, the dielectric constants appear to become independent of sample size suggesting that periodicity becomes less important as the system becomes more orientationally ordered. We note that the values for $N=32$ and $N=256$ (see tables 1 and 3 ) behave similarly.

In figure 2 we have attempted to explore the dependence of the integral dielectric constant upon field strength in more detail, as well as to investigate the influence of the boundary conditions. Values for $N=108$ and $\epsilon_{\mathrm{RF}}=15,85$, and $\infty$ are compared. We see in figure $2(a)$ that the boundary conditions appear to have a significant effect. However, it is clear from equations (2) and (4) that, whereas $\epsilon_{p}$ explicitly depends upon $\epsilon_{\mathrm{RF}}$ when viewed as a function of the applied field $E_{0}$, it should be independent of $\epsilon_{\mathrm{RF}}$ when $E_{0}$ is replaced by the Maxwell field $E$ (at least for macroscopic dielectrics). In figure $2(b)$, where $\epsilon_{p}$ is plotted against $E$, we observed that for larger fields $\epsilon_{p}$ does in fact become independent of the value of $\epsilon_{\mathrm{RF}}$. At smaller fields we find that the curves separate and, to within uncertainty, extrapolate to the appropriate zero-field results.

Equation (12) predicts that for sufficiently small fields, $\epsilon_{p}$ will have a simple quadratic dependence on $E_{0}$. In figure 2(c) we see that this claim is supported by 
P. G. Kusalik
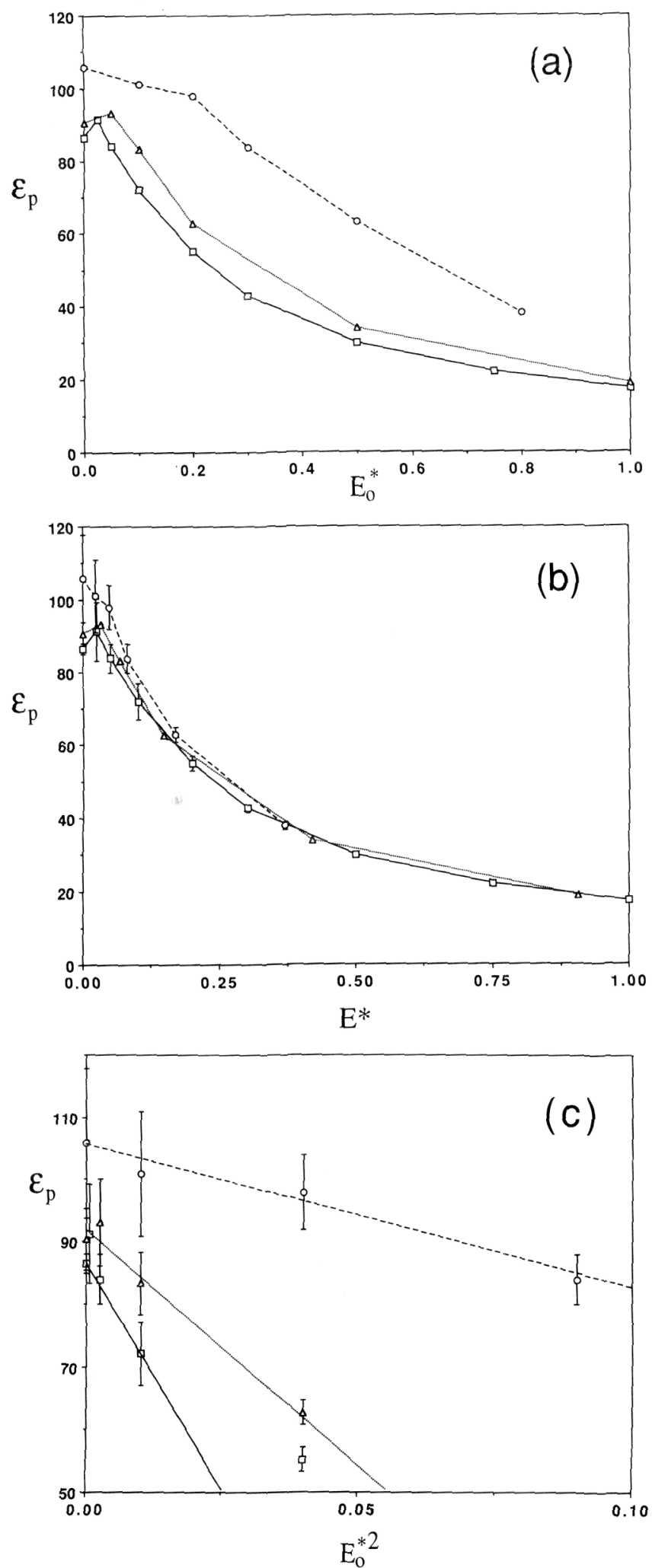

Figure 2. 
our data. Moreover, it is clear that the limiting slopes are all negative and that their magnitudes increase with the value of $\epsilon_{\mathrm{RF}}$. Unfortunately, a quantitative comparison (such as with equation (12)) would be difficult because of the large uncertainties at these smaller field strengths. Attempts were also made to exploit relationships such as equations (7) and (12) to determine the local field dependence of $\epsilon_{p}$ both at $E_{0}=0$ and at finite $E_{0}$. The statistical uncertainties in the higher moments of $M$ were at least as large as those for $\left\langle M^{2}\right\rangle$ and, with substantial numerical cancellation (see equations $(7 d-f))$ as a result of differencing two or more large values, the relative errors in $a_{2}$, $a_{3}$, etc. became prohibitive. Hence, even though we were able to provide the value for $a_{1}$ to within $5-10 \%$, we were unable to predict even the sign of $a_{3}$. A plot of $\left\langle M_{\|}\right\rangle$ against $E_{0}$ reveals that the slopes of the tangents to this curve usually vary quite significantly from one field point to the next (except at very small and very large fields). It is not surprising then that using equation ( $7 a$ ) to linear order only, we were not particularly successful at determining reasonably reliable estimates for $\left\langle M_{\|}\right\rangle$, and therefore $\epsilon_{p}$, from data accumulated at neighbouring fields. However, given a set of local slopes, $a_{1}$, we did have some success with integrating these values, knowing that they should satisfy the relationship

$$
\left\langle M_{\|}\right\rangle_{E_{0}}=\int_{0}^{E_{0}}\left(\frac{\partial\left\langle M_{\|}\right\rangle}{\partial E_{0}}\right)_{E_{0}^{\prime}} \mathrm{d} E_{0}^{\prime}
$$

to obtain reasonable estimates for $\epsilon_{p}$ at finite fields.

As mentioned above, another purpose of the present study was to examine electrostriction in this dipolar fluid. The previous work of Adams [15] using NpT Monte Carlo was inconclusive, due in part to poor convergence of the average densities. In figure 3 we have plotted data for the average densities from the present constant pressure molecular dynamics (MD) calculations for $N=500$. From thermodynamic arguments it is possible to show [21,27] that at sufficiently small fields the electrostriction observed for a polar liquid should vary as the square of the applied field. We see from the insert in figure 3 that this behaviour is clearly demonstrated by the present results, although only at the smallest fields considered. As the field strength increases, saturation effects are again exhibited. We remark that even at larger fields the total increase in density of this rather incompressible fluid is still only $1-1.5 \%$. In general, we would expect the electrostriction of the fluid to impact upon the nonlinear behaviour of its dielectric properties; however, for the present system these effects will obviously be small due to the relatively small changes in density observed. Short test calculations at constant volume $\left(\rho^{*}=0.8\right)$ and $E_{0}^{*}=0.3$ and 1.0 provided confirmation of this fact. As with the dielectric constants, when plotted against $E_{0}$ the density depends upon the value of $\epsilon_{\mathrm{RF}}$, yet if the Maxwell field is used this dependence virtually disappears (this is again the behaviour we would expect for a macroscopic dielectric [21,27]).

Plots similar to figure 3 have also been constructed for the average dipole-dipole energies reported in tables $1-4$. Once more we find an $E_{0}^{2}$ dependence at low fields and if the energies are plotted as functions of the Maxwell field the data at different values for $\epsilon_{\mathrm{RF}}$ collapse into a single curve.

Figure 2. The influence of $\epsilon_{\mathrm{RF}}$ upon the field dependence of $\epsilon_{p}$. The open squares, triangles and circles are, respectively, results from calculations at $\epsilon_{\mathrm{RF}}=\infty, 85$ and 15 . Shown is the dependence upon (a) the applied field, $(b)$ the Maxwell field, and $(c)$ the square of the applied field. Sample error bars have been included. The values at zero field have been taken from [9]. In $(c)$ the lines represent approximate limiting slopes. 


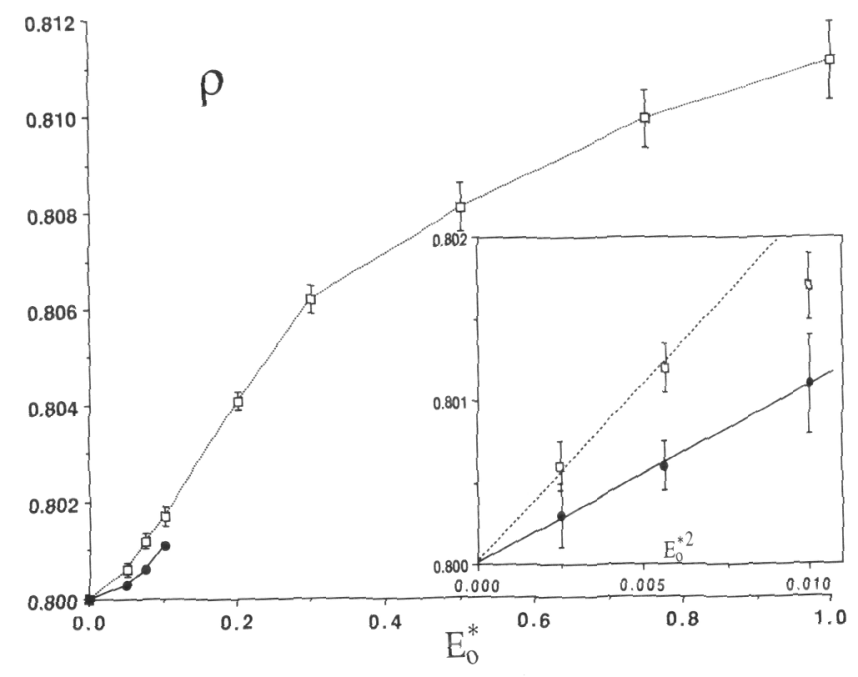

Figure 3. Density against applied field for a 500 particle sample. The dots are values recorded with $\epsilon_{\mathrm{RF}}=85$, while the open squares are points from calculations at $\epsilon_{\mathrm{RF}}=\infty$. The insert shows the dependence of the density upon the square of the applied field at small fields. Sample error bars have been included.

In a recent article Wei and Patey [28] have shown that with still larger dipole moments $\left(\mu^{*}=3 \cdot 0\right)$, these dipolar soft-sphere fluids can form ferroelectric nematic phases (with no applied fields). Although the present system $\left(\mu^{*}=2 \cdot 0\right)$ appears unable to form these orientationally ordered phases at zero field, we might nevertheless suspect that an applied field could stabilize such a phase. In an attempt to determine if a similar transition could be induced in the present dipolar soft-sphere fluid with the application of a sufficiently large applied electric field, several shorter calculations (typically 25000 timesteps) were carried out with samples of 108 particles at $\epsilon_{\mathrm{RF}}=\infty$ for fields up to $E_{0}^{*}=5$. However, examination of the average energies and densities revealed them to be smooth continuous functions (to within uncertainties) of the applied field. Since the system was more than $92 \%$ saturated at $E_{0}^{*}=5$, we would conclude that the dipolar coupling in the present dipolar softsphere fluid (with $\mu^{*}=2.0$ ) is not sufficient for it to demonstrate a nematic phase, even when exposed to extremely large electric fields.

\section{Conclusions}

In this paper we have examined the properties of a dipolar fluid in the presence of applied static homogeneous electric fields. In order to help facilitate the discussion of our simulation results for the dielectric response, we have summarized formal relationships for the differential dielectric constants, $\epsilon_{\|}$and $\epsilon_{\perp}$, and the integral dielectric constant, $\epsilon_{p}$. Molecular dynamics simulations have been performed at constant temperature and pressure for samples of 32, 108, 256 and 500 dipolar soft spheres at $T^{*}=1.35$ and $\mu^{*}=2.0$ for field strengths ranging from 0.025 to 1.0 . Previously, this system had been extensively studied at zero field $[9,19]$. The dependences of $\epsilon_{\gamma}, \epsilon_{1}$ and $\epsilon_{p}$ upon the applied field, as well as upon the sample size and boundary conditions applied, were explored. Electrostriction, the influence of the applied field upon the density of the system, was also investigated. 
In general, our results at smaller fields indicate that applied field simulations do not represent a computationally superior alternative to the usual equilibrium fluctuation approach to $\epsilon_{0}$. Although nonlinear effects do not become appreciable until the dipole saturation is more than about $20 \%$, the signal to noise ratios at low fields are also small making it rather difficult to extract precise values for the dielectric constants, particularly $\epsilon_{p}$. At small fields the influence of system size and boundary conditions upon $\epsilon_{\|}, \epsilon_{\perp}$ and $\epsilon_{p}$ was consistent with the behaviour previously detected in $\epsilon_{0}[9]$. However, at higher fields data from calculations with different numbers of particles and values for $\epsilon_{\mathrm{RF}}$ were found to lie on a single curve when viewed as functions of the Maxwell field. We were able to demonstrate an apparent $E_{0}^{2}$ dependence in $\epsilon_{p}$ at low fields; yet it was not possible to make a comparison between estimates for this slope given by the appropriate statistical mechanical expressions (see equations (7) and (12)) and the measured data due to very large uncertainties (in excess of $100 \%$ ) in the former. At all non-zero fields it was observed that the values for $\epsilon_{\|}$measuring the differential reponse parallel to the applied field were always less than those for $\epsilon_{\perp}$. At the same time the results for the differential dielectric constant, $\epsilon_{1}$, were discovered to be always equal (to within uncertainties) to those of the integral dielectric constant, $\epsilon_{p}$, although we are not aware of any formal justification for this rather surprising behaviour.

Electrostriction within our dipolar soft-sphere fluid was also monitored. At small fields the increase in density exhibited the expected $E_{0}^{2}$ dependence, although saturation effects again become apparent when the polarization reaches approximately $25-30 \%$ of its maximum value.

A secondary aspect of the present study was an attempt to induce (with the application of a sufficiently large electric field) a nematic phase similar to that observed by Wei and Patey [28] for dipolar soft-sphere systems with larger dipole moments. Although we have subjected the present fluid to very high electric fields, no anomalous behaviour was detected in any of the properties examined. Hence, we conclude that the dipole couplings are not sufficient in the present system to support a nematic phase even in the presence of extremely large fields.

Clearly, the full time-dependent dielectric response of polar liquids would be required if a much more complete picture of dynamical processes in these systems is desired. Thus, we have also carried out MD simulations in which time-dependent (oscillating) electric fields have been applied to the dipolar fluid studied here. This investigation will be the subject of a future article [29].

We are grateful for the financial support of the Natural Sciences and Engineering Research Council of Canada.

\section{References}

[1] Buckingham, A. D., 1955, J. chem. Phys., 25, 428.

[2] Kolodziej, H. A., Parry Jones, G., and Davies, Mansel, 1975, Trans. Faraday Soc. II, 71, 269.

[3] Patey, G. N., Levesque, D., and Weis, J. J., 1982, Molec. Phys., 45, 733.

[4] de Leeuw, S. W., Perram, J. W., and Smith, E. R., 1980, Proc. R. Soc. A, 373, 27; 1983, Ibid., A388, 177.

[5] Neumann, M., and Steinhauser, O., 1983, Chem. Phys. Lett., 95, 417.

[6] Neumann, M., 1986, Molec. Phys., 57, 97.

[7] Neumann, M., Steinhauser, O., and Pawley, G. S., 1984, Molec. Phys., 52, 97. 
[8] Neumann, M., 1983, Molec. Phys., 50, 841.

[9] Kusalik, P. G., 1990, J. chem. Phys., 93, 3520.

[10] de Leeuw, S. W., Perram, J. W., and Smith, E. R., 1986, Ann. Rev. Phys. Chem., 37, 245.

[11] Neumann, Martin, 1985, J. chem. Phys., 82, 5663; 1986, Ibid., 85, 1567.

[12] Levesque, D., and Weis, J. J., 1984, Physica A, 125, 270.

[13] (a) Alder, B. J., and Pollock, E. L., 1981, Ann. Rev. Phys. Chem., 32, 311; (b) Pollock, E. L., and Alder, B. J., 1980, Physica A, 102, 1.

[14] Adams, D. J., 1980, Molec. Phys, 40, 1261.

[15] Adams, D. J., and Adams, E. M., 1981, Molec. Phys., 42, 907.

[16] Alper, H. E., and Levy, R. M., 1989, J. chem. Phys., 91, 1242.

[17] Petersen, H. G., de Leeuw, S. W., and Perram, J. W., 1989, Molec. Phys, 66, 637.

[18] Kusalik, P. G., 1992, Molec. Phys, , 76, 337.

[19] Kusalik, P. G., 1991, Molec. Phys., 73, 1349.

[20] Coffey, W. T., and Scaife, B. K. P., 1976, Proc. R. Irish Acad. A, 67, 195.

[21] Böttcher, C. J. F., 1973, Theory of Electric Polarization, second edition, Vol. 1 (Elsevier Scientific).

[22] Fröhlich, H., 1958, Theory of Dielectrics (Oxford University Press).

[23] Evans, D. J., and Morriss, G. P., 1990, Statistical Mechanics of Nonequilibrium Liquids (San Diego: Academic).

[24] Allen, M. P., and Throeslex, D. J., 1989, Computer Simulation af Ligujids (Oxford. Clarendon Press).

[25] Nosé, S., 1984, J. Chem. Phys., 81, 511; 1984, Molec. Phys., 52, 255.

[26] Evans, D. J., 1977, Molec. Phys., 34, 317.

[27] Stenschke, H., 1966, Physical Chemistry: An Advanced Treatsie, vol. 1, edited by H. Eyring, chap. 5.

[28] Wei, Dongquing and Patey, G. N., 1992, Phys. Rev. Lett., 68, 2043.

[29] Kusalik, P. G., 1993, (to be published). 